\title{
AN EMPERICAL CASE RESPONSE TO DOES INFORMATION SYSTEMS SUFFER AN IDENTITY CRISIS? A CASE STUDY OF CONFUSION AND MISINFORMATION
}

\author{
Anthony Rodi, D.Sc., California University of Pennsylvania, rodi@calu.edu \\ Gina M. Boff. D.Sc., California University of Pennsylvania, boff@calu.edu \\ Gary Delorenzo,D.Sc., California University of Pennsylvania, delorenzo@calu.edu
}

\begin{abstract}
At IACIS 2012, a case study was presented that explored the CIS identity crisis occurring at a 9,000-student, state institution in the northeast, U.S.A. Confusion, miscommunication, and an ignorance of - and a general lack of respect for - the CIS discipline were identified as some of the reasons behind the underrepresentation of CIS at that university, resulting in suffering enrollments in the major. In direct follow up to that effort, this paper explores some empirical case evidence of this same "CIS Invisibility Phenomenon" at the northeastern institution, in attempts to drill down to the fundamental pitfalls in the promotion of CIS at that university. While research shows that there is no silver-bullet solution to this prominent and on-going issue in CIS discipline recognition, the continuation of this study could prove beneficial in understanding the cultural implications of the phenomenon and offer some insight to counter the residual, negative effects.
\end{abstract}

Keywords: Information Systems, Computer Information Systems, IS, CIS, and CIS Curriculum

\section{INTRODUCTION AND LITERATURE REVIEW}

The inscrutable evolution of computer-based information systems (Computer Information Systems (CIS), also commonly referred to as Information Systems (IS)), set the stage for the seemingly endless debate concerning its boundaries and its up-hill quest for respect from its science-focused predecessors. The following excerpt from Gordon. Davis' [7] forward in Information Systems: The State of the Field offers a succinct description of CIS' formation:

Information systems as an academic field did not spring forth full grown. It emerged slowly from the mid1950s and through the 1960s. During this period, a relatively small number of professors at universities in different countries explored the use of computers for processing and storing data in organizations. These professors had a variety of academic backgrounds such as accounting, organizational behavior, management, operations, management science and economics. These diverse backgrounds, when focused on information systems in organizations, were brought together in forming a field of study in information systems (under a variety of names). Using the late 1960 s as the starting point for the field, it is less than 40 years old. Among the organization disciplines, we are the youngest. [7].

Yes; "we are the youngest"; however, 40 years is not a trivial amount of time for the establishment of a discipline. Take for example the speed at which new avenues of research in the medical field (also a "practitioner" field, as CIS is often referred with negative connotation) birth fully-respected and recognized specializations resulting in a continuous increase in its academic disciplines. [12, 15]. Yet, with CIS, it was not until the late 1990's that the CIS discipline seemed to finally make a breakthrough when it was established as a "reference discipline" by a good number of established scholars $[4,24]$, only to be met with more admonishment from its computing-related peers and immediate efforts to repudiate the claim. [25].

Today, in 2015, the debate ensues. A quick review of current literature will find computing scholars still debating, defining, disparaging and defending the CIS discipline. So perhaps it should come as no surprise that the "confusion, misinformation and disrespect" identified in the initial case, transcends literature and permeates the academic culture of universities [16]. And even in that collection of treatise, the acronyms MIS, IS, IT and CIS are often all used interchangeably by scholars in the CIS field! [5, 10]. There are a number of reasons for this - all of 
which are intricately connected; these are: the interdisciplinary nature of CIS, the "IT artifact", the "sub-par" classification of business journals to the scientific community, and the negative connotation of that same community to "practitioner" fields $[16,13,11]$. Each of these will be discussed within the boundary of a computer information system, as defined by its purpose.

We accept the principle purpose of any type of business information system as providing employees, at any level and in any position within an organization, optimized information that enables those employees to make the best possible decisions in carrying out their daily work in the most productive manner possible. While it is entirely conceivable to bring this about with a non-technologically based solution, such as an adjustment of workflow through a business process redesign, in most cases, there is some form of a computing-based technology to assist in the process optimization and information dissemination. [17]. This technological component necessary in a computer-based information system brings us to the concept of "The IT Artifact" [19, 26].

\section{The "IT Artifact"}

The necessary technological component of a computer-based information system is commonly referred to in the literature as the "IT artifact" $[19,26]$. Its necessary inclusion in a computer-based information system mandates that the IT artifact remain in the scope of the CIS discipline - in practice, in curriculum studies, and in areas of research and publications. And as such, the IT artifact causes the lines of the CIS discipline to become blurred with the strictly technological field of Information Technology.

What's more is that the IT artifact, itself, can range in sophistication from the simplest alteration of a legacy computer system to the most cutting-edge, satellite-bouncing mobile technologies available today, marking it as a sister-discipline to CIS that is lacking in focus and producing, what many scholars consider to be, sub-par research contributions. All of these aspects will be discussed.

\section{The Interdisciplinary Nature of the CIS Field}

By its very nature, CIS as an inter-disciplinary field of study and would not be in existence if it didn't pull together, at a minimum, the areas of Business, Computer Science and/or Information Technology, and a variety of the Social Sciences [1]. A corporation's competitive edge is gained through superior choices in business decision making [21]. Those choices are enabled through the right information, to the right people, in the right time frame. It's that simple. And that optimal information dissemination starts with streamlined computer information systems that are integrated from the shop floor through to the data warehouse used by key executives [17]. Bringing such an infrastructure into existence is no trivial task and is accomplished only through the well-versed professionals in the field of CIS. It is not strictly business savvy, as current technology is the enabler. It is not technical savvy, as a deep understanding of business operations at each and every corporate level is required. And it's not just the combination of the latter two, as organizations are naturally resistant to the continual changes required to keep competitive pace; and so the CIS professional must have keen organizational psychology, interpersonal, communication, and management skills as well [1].

This interdisciplinary construct of CIS, which is not only the virtue of the discipline but also the strength of the respective organization in which it is housed, is its Achilles heel in attaining recognition and respect in the academic and scholarly community. CIS is considered "fragmented" and "lacking focus" [11]. The CIS reference disciplines of Business, Computer Science and/or Information Technology, and Social Science do not share an orientation in their activities and research methodologies, and the social science aspect, itself, is "marked by a plethora of schools of thought, each with its own meta-theoretic assumptions, research methodologies and adherents" [1, orig. 19]. This leads to two things: CIS having the air of being overly pragmatic, and thus lacking the investigative rigor found in scientific theory; and, a varied journal community for CIS publications, lending itself to a perception of sub-par research and contributions. Both of these are discussed. 


\section{CIS as a "Practitioner" Field}

CIS is not - and cannot be - consistent without sacrificing its great effectiveness, as one of its largest strengths lie in "the creativity and innovative effort" that is applied in business solutions [1]. And yet, this "application" component to CIS, which is, again, a key construct of the field, is another source of ridicule from academics and scholars as it is pegged into a lower classification of "practitioner" field, weighed down with pragmatism, as opposed to a traditional "theoretical" area of study [6]. This paper's authors boldly counter this negative connotation associated to applied, practitioner fields with this rhetorical question: "and what, exactly, is the field of medicine?" As cited in Weber, well-versed scholars have argued that "a call for unifying the paradigm is not desirable"; we agree. [orig. 2, orig. $11]$.

\section{Research Contributions Perceived "Sub-Par"}

With its roots in Computer Science, the CIS discipline uses a variety of social science skills to analyze business processes and identify areas of improvement. Through creativity and ingenuity, processes are optimized and further streamlined by incorporating an Information Technology component that is integrated into the organization by, again, engaging the skills of social science. The solution is not theoretical; it is a physical change and implementation, which marks it as the result of a practitioner, and not that of a scientist.

The necessary inclusion of the IT artifact, plagued with its own natural diversity, the problem space of CIS defined as business, and the ethereal, yet quite necessary employment of social sciences to define solutions and bring them to fruition, leave the potential research and publication outlet options broader than the traditions associated to sciences will permit.

What has resulted is the characterization of CIS as "fragmented adhocracy - a discipline characterized by a low level of political independence among its members, a low level of conceptual coherence, and a low level of coherence in terms of its standardization of research methods and interpretation of results" [26, orig. 2).

CIS is not accepted is a pure participant in any of its reference disciplines (Business, IT, Computer Science and Social Sciences) and so the CIS contributions to the existing body of knowledge have no, one, home in the research community, which leads to the final misconception discussed here; and that is, that this diversity in research concepts, methods, and journal communities, gives the perception of sub-standard research contributions.

Studies conducted to examine the publication outlet availability in, what are classified as elite journals, found significantly less CIS publications by CIS faculty and professionals [8]. The reason behind this, in summation, is that each of the previously-discussed concepts weave together an erroneous perception of the CIS discipline among academics and scholars - the population who carry over this misconception into their areas of profession: the university community.

\section{Cultural Carry-Over to Academic and Scholarly Communities}

Each university has its own culture, with each school housed therein bearing its own sub-culture. [20]. Much of the construct of the manner of social contracts and the way business is done in academia is heavily influenced by tradition and convention, some of which have been carried through time as far back as the days of Socrates [23].

Individuals carry bits of their cultural influences with them when they traverse into other cultures and often, whether intentionally or not, instigate a struggle with the status quo. Newcomers behave in ways to influence the existing environment which is met with resistance from the established to maintain a cultural equilibrium. And such is the on-going tug-of-war between the older disciplines of highly-mathematical, theoretical roots of Computer Science and Engineering, with its relatively new-comer of Computer Information Systems, and the internal stakeholders associated to each [11]. This erroneous perception of CIS from the research and publication community being carried over into the university internal stakeholder forum is quite costly to the discipline as key players in the academic business paradigm, acting under misinformation, make poor decisions as they relate to CIS programs and majors. 


\section{THE CATALYST TO INQUIRY}

The original 2012 case analysis of this university, from Kohun et al [16], was based on the ethnographic observations of the researchers, living and working in the ominous academic culture wherein CIS is slighted. After comparing the curricular contents of three computing programs (Computer Science, Management Information Systems and Computer Information Systems), a qualitative discussion ensued to give light to their educated suspicions as to the potential motives behind the CIS identity crisis at the university, which included, in part:

Computer Science

- $\quad$ Considered a true science and technically based discipline

- $\quad$ More hard-skill oriented in the areas of engineering, software design based on mathematical algorithms

- $\quad$ CS faculty has the more challenging skill sets needed for success in the marketplace

- Scientific research and inquiry - publications in scientific journals - peers of engineering-types

(Computer) Information Systems

- $\quad$ Considered a generalist program where people use technology along the way

- $\quad$ More soft-skill oriented in problem solving and decision making while using some technology based solutions along the way.

- CIS faculty do not have the intellectual capacity of the CS faculty

- $\quad$ Social science research and inquiry - publications in business and MIS journals - peers are diverse

That team of researchers pointed to some of the negative effects to the CIS program that are resulting from poor decisions being made, as relates to CIS, due to ignorance of the discipline and misinformation brought about by these erroneous perceptions, including lack of marketing, misallocation of funding, and the ill advisement of incoming-freshmen leading to low enrollment.

While that publication offered the deep and rich insights that can only be gained from lengthy experience with the subject, no empirical evidence could be presented at that time. In an effort to potentially quantify some of those initial assertions, this research team began to make formal data requests through university administration and other reporting request venues, to collect both the student enrollment and student graduation statistics of the two programs exhibiting the highest contention: Computer Information systems (CIS) and Computer Science.

Research shows that this CIS issue is not restricted to this one specific case; but instead, exists in nearly all universities that have a program/major in CIS, due to this cultural carry-over. The empirical evidence uncovered in this 2015 case not only proves the initial assumptions correct, but it also gives light to the underpinnings as to why CIS suffers at the hand of social contracts and misconception in academia.

\section{METHODOLOGY}

Enrollment data for both CIS majors and CS majors was collected from 2005 to 2015 from the university's student registration system, capturing a total of 881 non-recurring records, which included:

\begin{tabular}{|c|c|c|c|c|c|c|}
\hline $\begin{array}{l}\text { Non-Identifying } \\
\text { Student Number }\end{array}$ & $\begin{array}{l}\text { Current } \\
\text { Semester and Year }\end{array}$ & $\begin{array}{l}\text { Major } \\
\text { at Present }\end{array}$ & $\begin{array}{l}\text { Entry to University } \\
\text { Semester and Year }\end{array}$ & $\begin{array}{l}\text { Status at Entry } \\
\text { Incoming Freshmen or Transfer }\end{array}$ & $\begin{array}{l}\text { Major } \\
\text { at Entry }\end{array}$ & $\begin{array}{l}\text { Graduation } \\
\text { Date }\end{array}$ \\
\hline
\end{tabular}

In addition, an enrollment total was calculated, by semester (Spring, Summer, Fall). This allowed for a number of comparisons beyond the typical annual enrollment comparison of the two majors. Without even running any statistics, the initial analysis uncovered clear trends in student enrollment in these two programs.

\section{Data Analysis Methods}

The quantitative enrollment data was collected, summarized and was analyzed using Microsoft Excel to provide a comparison of enrollment trends for both CIS and CS programs from Fall 2005 to Spring 2015. The CIS program 
was launched in Fall 2005, so we used comparative data with CS starting that same year. Analysis of the data was then conducted to determine internal transfer of students from CS to CIS as a change of major.

\section{FINDINGS AND DISCUSSION}

\section{Initial Enrollment: CS vs CIS}

Both Table 1 and Figure 1 depict the number of students enrolled in each respective program, from the Fall 2005 through the Spring 2015, in aggregated totals for students enrolled for fall, spring and summer terms:

Table 1. Initial Enrollment of CS vs CIS

\begin{tabular}{|lcrc|}
\hline \multicolumn{1}{|c}{ Years } & Comp Info Sys & Comp Sci & Grand Total \\
\hline $2005-2006$ & 10 & 144 & 154 \\
$2006-2007$ & 38 & 73 & 111 \\
$2007-2008$ & 46 & 84 & 130 \\
$2008-2009$ & 53 & 147 & 200 \\
$2009-2010$ & 71 & 157 & 228 \\
$2010-2011$ & 73 & 123 & 196 \\
$2011-2012$ & 74 & 125 & 199 \\
$2012-2013$ & 81 & 118 & 199 \\
$2013-2014$ & 82 & 101 & 183 \\
$2014-2015$ & 77 & 100 & 177 \\
Grand Total & 605 & 1172 & 1777 \\
\hline
\end{tabular}

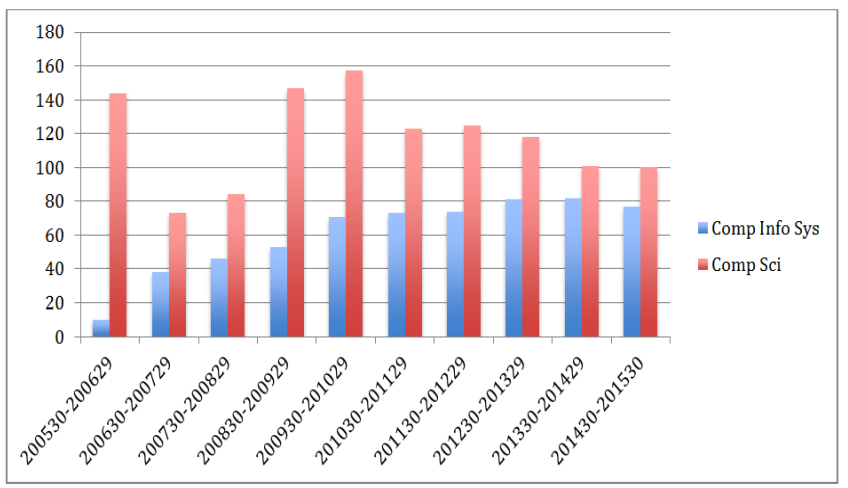

Figure 1. Initial Enrollment of CS vs CIS

Analysis of this data, as seen depicted in the charts, returned expected results. The enrollment data shown in Table1 and Figure-1 both show that the CS program had higher enrollments due to its being an established, known program, as the CIS program began in Fall 2005, in the shadow of the long-established CS program. The results reflect the newness of the CIS discipline in 2005 with an enrollment of 10 students, to enrollment of the current 2014-2015 academic year of 77 students.

\section{Transfer/Admit Analysis}

Next, the Transfer /Admit Trend data was analyzed to determine the transfer rate of CS as compared to CIS programs. Shown in Table 2 below is the data reflecting the Transfer/Admit Trend data for CIS vs CS from Fall 2005 through Spring 2015. The data reflects aggregated totals for students who transferred into respective programs (CS, CIS) from outside of the university during fall, spring and summer terms. The following chart in Figure 2 is a graphical representation of this, same, Transfer/Admit data.

Table 2. Transfer/Admit Trends

\begin{tabular}{|cccc|}
\hline Years & Comp Info Sys & Comp Sci & Grand Total \\
\hline $2005-2006$ & 9 & 20 & 29 \\
$2006-2007$ & 24 & 30 & 54 \\
$2007-2008$ & 26 & 20 & 46 \\
$2008-2009$ & 16 & 21 & 37 \\
$2009-2010$ & 25 & 31 & 56 \\
$2010-2011$ & 26 & 26 & 52 \\
$2011-2012$ & 39 & 40 & 79 \\
$2012-2013$ & 28 & 27 & 55 \\
$2013-2014$ & 21 & 20 & 41 \\
$2014-2015$ & 7 & 15 & 22 \\
Grand Total & 221 & 250 & 471 \\
\hline
\end{tabular}

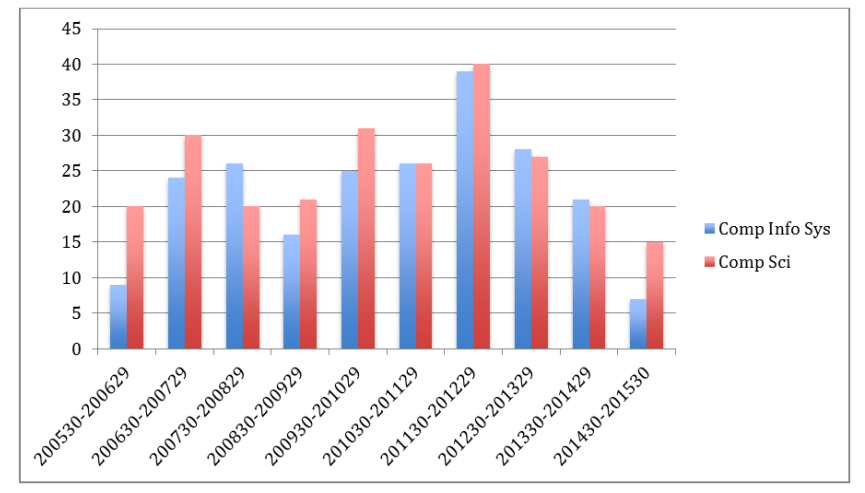

Figure 2. Graph Analysis of Transfer/Admit Trends 
Based on previous knowledge of transfer/admittance of students into CS and CIS, an analysis of data reflecting incoming students from outside the university into the CIS program from Fall 2005 through Spring 2015 was conducted. These students are usually an external transfer as a result of a change of universities or new incoming students. A higher transfer rate into CIS vs. CS was expected; however, the data shows no significant difference I the number of transfers, from external sources, into either program.

\section{Internal Transfers from CS to CIS}

Data collected and presented here, in Table-3 and Figure 3 below, shows the number of students enrolled in Computer science (CS) that transferred to Computer Information Systems (CIS):

Table 3. CS to CIS Transfers

\begin{tabular}{|cc|}
\hline School Year & CS to CIS Transfers \\
\hline 2005 to 2006 & 0 \\
2006 to 2007 & 9 \\
2007 to 2008 & 2 \\
2008 to 2009 & 2 \\
2009 to 2010 & 7 \\
2010 to 2011 & 3 \\
2011 to 2012 & 5 \\
2012 to 2013 & 8 \\
2013 to 2014 & 5 \\
2014 to 2015 & 6 \\
\hline Total & 47 \\
\hline
\end{tabular}

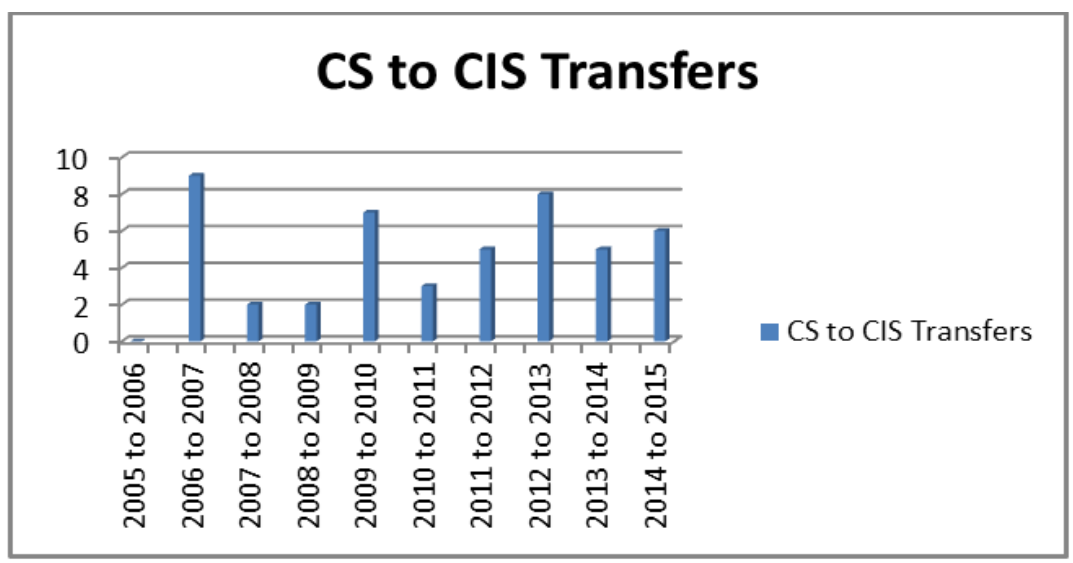

Figure 3. Graph Analysis of CS to CIS Transfers

Shown in Table 3 is the data reflecting the CS to CIS Transfer students from Fall 2005 through Spring 2015. The data reflects totals for students who transferred into respective programs during fall, spring and summer terms. The chart in Figure 3 is a graphical representation of the CS to CIS Transfer data.

This constitutes the most interesting trend discovered - the significant number of students who started in the CS program and transferred to CIS shortly thereafter. There was an initial assumption, on the part of the researchers, that a certain number of incoming students enrolled in the CS program did not intend to stay in that program for a number of reasons. What the data showed was that $47 \mathrm{CS}$ students had changed their major to CIS as a result of becoming more knowledgeable and informed of the CIS program.

What is occurring is that incoming freshmen are entering Computer Science with little to no knowledge of the Computer Information Systems program, which is in the same department. Once a student is in the CS program for a while and is exposed to other programs in the university, either through conversations with CIS students, inquiries, or through their own curiosity, they change their major to CIS, which more closely aligns with their original academic goals as incoming freshmen.

\section{Transfers from Other Majors to CIS}

The final analysis was done to determine the number of students from other majors within the university, who transferred into the CIS major. The student transfer data are shown in Table 4 and Figure 4 as indicated in the table and chart below: 
Table 4. Other Majors to CIS

\begin{tabular}{|c|c|}
\hline Academic Year & $\begin{array}{c}\text { Other Majors } \\
\text { Transfer to CIS }\end{array}$ \\
\hline 2005 to 2006 & 4 \\
2006 to 2007 & 24 \\
2007 to 2008 & 17 \\
2008 to 2009 & 14 \\
2009 to 2010 & 17 \\
2010 to 2011 & 16 \\
2011 to 2012 & 22 \\
2012 to 2013 & 19 \\
2013 to 2014 & 17 \\
2014 to 2015 & 4 \\
\hline Total & 154 \\
\hline
\end{tabular}

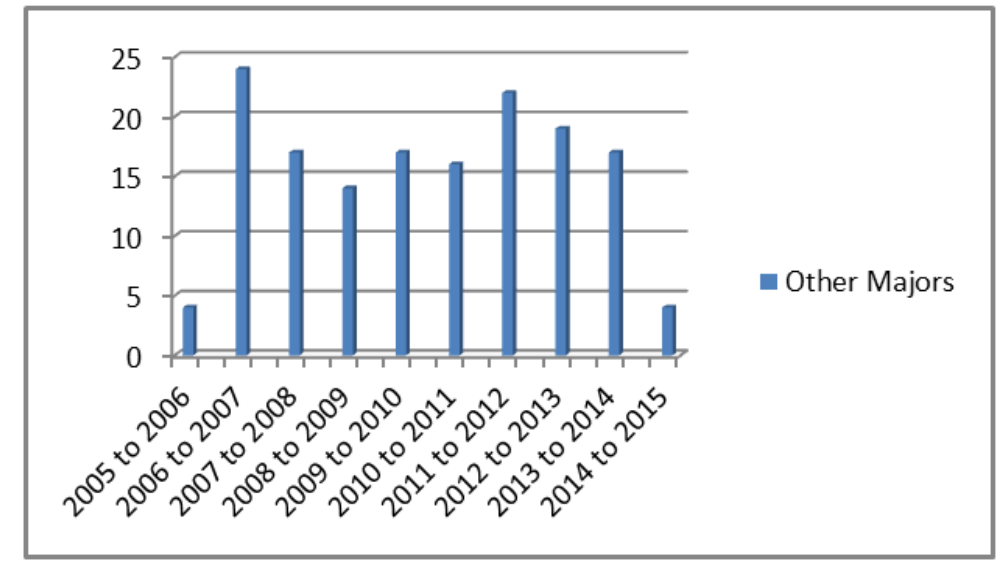

Figure 4. Graph Analysis of Other Majors to CIS

Shown in Table 4 is the data reflecting other majors to CIS transfer students from Fall 2005 through Spring 2015. The data reflects totals for students who transferred into respective programs during fall, spring and summer terms. The chart in Figure-4 is a graphical representation of other majors to CIS Transfer data.

Note that from the Fall 2005 to Spring 2015, there have been 154 internal transfer students who left their major and declared CIS as their major. It can only be deduced that this is due to the continued lack of information - or misinformation - given to incoming freshmen concerning their option to major in CIS.

\section{OTHER DISCUSSION}

What was discovered was lower enrollment in the discipline, at this university, as a strict result of the misinformed actions of internal stakeholders. The CIS program exists in the same department and CS and still lives in its shadow, causing an ongoing identity crisis. The research proves that there is interest in the CIS discipline once it is discovered. In addition, what was also discovered as the data was collected was that some students do not declare their major or change of major in the summer, but wait until early fall to make the change.

As a result, CIS is not easily recognized or respected; therefore, is not promoted properly through marketing or other means. And they are unknown because they are not recognized as a stand-alone discipline. The lack of knowledge prohibits enrollment growth, and the lack of enrollment growth leaves them unfunded or underfunded. Programs/majors in CIS suffer, in terms of success and sustainability, as a result of the financial implications of the cultural carryover of erroneous perceptions from the research and publication community.

\section{Incoming Freshmen - Limited Understanding of CIS}

A lack of understanding the CIS discipline throughout the university is the culprit, especially with the admissions function of the university. Instead of taking the time to learn about the disciplines in which they take charge, it is easier for them to just go with the flow of the culture, which is erroneously influenced by the professors that work in it. CIS struggles to be recognized at almost every function, meeting or activity, when CS, who is in the same department, is continuously mentioned.

Incoming freshmen are counseled in majors by admissions, guidance counselors, and teachers, and as soon as they hear the word "computing" from the student and/or parent, they are automatically pointed toward CS. For example, when working various Open House events on campus, it was discovered that students who were guided to the department with the thought of becoming a CS major actually discovered that they aligned better with the CIS 
discipline. Many students don't choose [CIS] as a major due to the perceptions about the work, opportunity, and educational curriculum - reasons quoted for this are the collapse of .com, off-shoring giving the perception of a lack of jobs, and ERP systems replacing jobs [10,3].

The reality is that once students understand the disciplines of both CS and CIS, they transfer into CIS program. The problem posed by this realization is the transfer to CIS may involve loss of time and credits towards the degree. "Some schools have focused on educating the high school counselors, parents and students about the field of MIS and explaining how MIS differs from Computer Science, hoping to inform potential freshmen that there is a tremendous difference between MIS and CS programs [9].

\section{CONCLUSIONS}

CIS is a best of breed discipline, pulling what works best from business and social sciences, and disbanding the outdated components of its roots in Computer Science to put to task what works in today's technological business solutions. It is generally misunderstood compared to Computer Science and Management Information Systems, yet it is a highly marketable discipline. Its broad appeal makes the traditional sciences - particularly that of its highly mathematical, theoretical predecessor of Computer Science, uneasy in their acceptance of CIS literature in traditional paradigms. This unacceptance carries over to the culture of the university, where administration continues to overlook CIS' value, in terms of funding and promotion with students. Under this premise, this study continued to explore the case of a northeast, state institution, started in 2012, where the initial researchers recognized the unacceptance of CIS as a respected discipline and reported qualitative deductions, but where no empirical evidence could be presented. The data collected through this effort shows the continued existence of the "CIS Invisibility Phenomenon" at the university, through a large number of incoming freshmen starting in Computer Science, which transfer to CIS upon starting their schooling. It also shows a higher ratio of students succeeding in CIS than CS, despite the high numbers of CS freshmen. This is attributed to the continued underrepresentation of CIS as a promising and respected discipline, which is a result of the stigma placed on CIS by the academic community. Finally, the data shows an extreme number of incoming transfers, from other universities into the CIS program as opposed to the CS program, where students became aware of the viability of the discipline through their own investigation and experience and are not dependent upon university administrators to guide them to the proper computing discipline best suited to their personal and professional goals.

Future research on this endeavor will include discussion with administrators, especially with the admissions function at the university, on improving the perception of the CIS program. A collaborative effort is needed to educate the university in such a way as to influence cultural change to accept and promote the value-added by the CIS discipline and its promise to students to attain unique business, technology and critical thinking skills that are highly marketable in the workforce.

\section{REFERENCES}

1. Avison, D., Elliot, S. (2006). Scoping the discipline of information systems. Information Systems: The State of the Field, 3-18

2. Banville, C., Landry, M. (1989). Can the Field of MIS Be Disciplined? Communications of the ACM (32:1), pp. 48-60.

3. Barnes, C., \& Bandyopadhyay, K. (2012). A successful curriculum strategy to increase MIS enrollment. Proceedings of the Southwestern DSI.

4. Baskerville, R. and M. Myers (2002) "Information Systems as a Reference Discipline", MIS Quarterly, (26)1, pp. 1-14.

5. Benbasat, I. and R. Zmud (2003) "The Identity Crisis Within the IS Discipline: Defining and Communicating the Discipline's Core Properties," MIS Quarterly, (27)2, pp.183-194.

6. Constantinides, P., Chiasson, M. W., \& Introna, L. D. (2012). The ends of Information Systems research: A pragmatic framework. Mis Quarterly, 36(1), 1-20.

7. Davis, G.B. (2006). Forward. In King, J. L., \& Lyytinen, K. (Eds.) Information systems: The state of the field (pp.xvii-xxi). Chichester, UK: John Wiley \& Sons.

8. Dean, D. L., Lowry, P. B., \& Humpherys, S. (2011). Profiling the Research Productivity of Tenured Information Systems Faculty at US Institutions. MIS Quarterly, 35(1), 1-15. 
9. Downey, J., McGaughey, R., \& Roach, D. (2011). Attitudes and influences toward choosing a business major: The case of information systems. Journal of Information Technology Education: Research, 10(1), 231-251.

10. George, J. F., Valacich, J. S., \& Valor, J. (2005). Does information systems still matter? Lessons for a maturing discipline. Communications of the association for information systems, 16(1), 8.

11. Hirschheim, R., Klein, H., Lyytinen, K.(1996). Exploring the Intellectual Structures of Information Systems Development: A Social Action Theoretic Perspective, Accounting, Management and Information Technologies (6:1/2), pp. 1-64.

12. History of Medicine Timeline. (n.d.). Retrieved February 14, 2015, from http://www.datesandevents.org/eventstimelines/10-history-of-medicine-timeline.htm

13. King, J. L., Lyytinen, K. Introduction (Eds.). (2006). Introduction. In Information systems: The state of the field (pp.xxiv-xxvi). Chichester, UK: John Wiley \& Sons.

14. Klein, H. K. (2003). Crisis in the IS Field? A Critical Reflection on the State of the Discipline. Journal of the Association for Information Systems, 4(1), 10.

15. Klein, H.K., Hirschheim, R.A. (2006). Information systems: The state of the field (pp.71-130). Chichester, UK: John Wiley \& Sons.

16. Kohun, F.G., Rodi, A.F., DeLorenzo, G.J.(2012). Does information systems suffer an identity crisis? A case study of confusion and misinformation. Issues in Information Systems, Volume 13, No.2, 2012

17. Monk, E., Wagner, B. (2012). Concepts in enterprise resource planning. Cengage Learning.

18. Orlikowski, W. J., Iacono, C. S. (2001). Research commentary: Desperately seeking the "IT" in IT researchA call to theorizing the IT artifact. Information systems research, 12(2), pp. 121-134.

19. Orlikowski, W.J., Baroudi, J.J. (1991). Studying information technology in organizations: Research approaches and assumptions Information systems research, 2(1), pp.1-28.

20. Sporn, B. (1996). Managing university culture: an analysis of the relationship between institutional culture and management approaches. Higher Education, 32(1), 41-61.

21. Stair, R (2013). Principles of Information Systems, $9^{\text {th }}$ Edition. Pearson Publishing.

22. Trevitt, C. (2008). Learning in academia is more than academic learning: action research in academic practice for and with medical academics. Educational Action Research, 16(4), 495-515.

23. Vaisey, S. (2008, September). Socrates, Skinner, and Aristotle: Three Ways of Thinking About Culture in Action1. In Sociological Forum (Vol. 23, No. 3, pp. 603-613). Blackwell Publishing Ltd.

24. Vessey, I., V. Ramesh, and R. Glass (2002) "Research In Information Systems: An Empirical Study Of Diversity In The Discipline And Its Journals," Journal of MIS, (19)2, pp. 129-174.

25. Wade, M., Biehl, M., \& Kim, H. (2006). Information systems is not a reference discipline (and what we can do about it). Journal of the Association for Information Systems, 7(1), 14.

26. Weber, R. (2003). Still desperately seeking the IT artifact. MIS quarterly, 27(2), 183-183. 